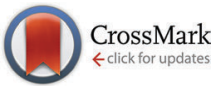

Cite this: Phys. Chem. Chem. Phys.,

Received 20th May 2016, Accepted 11th August 2016

DOI: $10.1039 / \mathrm{c} 6 \mathrm{cp} 03462 d$

www.rsc.org/pccp 2016, 18, 24654

\section{Spectroscopy and dynamics of double proton transfer in formic acid dimer $\dagger$}

\author{
Kasper Mackeprang, ${ }^{a}$ Zhen-Hao Xu, ${ }^{b}$ Zeina Maroun, ${ }^{a}$ Markus Meuwly* ${ }^{\star b}$ and \\ Henrik G. Kjaergaard*a
}

\begin{abstract}
We present the isolated gas phase infrared spectra of formic acid dimer, $(\mathrm{HCOOH})_{2}$, and its deuterated counterpart formic-d acid, $(\mathrm{DCOOH})_{2}$, at room temperature. The formic acid dimer spectrum was obtained by spectral subtraction of a spectrum of formic acid vapor recorded at low pressure from that recorded at a higher pressure. The spectra of formic acid vapor contain features from both formic acid monomer and formic acid dimer, but at low and high pressures of formic acid, the equilibrium is pushed towards the monomer and dimer, respectively. A similar approach was used for the formic-d acid dimer. Building on the previous development of the Molecular Mechanics with Proton Transfer (MMPT) force field for simulating proton transfer reactions, molecular dynamics (MD) simulations were carried out to interpret the experimental spectra in the $\mathrm{OH}$-stretching region. Within the framework of MMPT, a combination of symmetric single and double minimum potential energy surfaces (PESs) provides a good description of the double proton transfer PES. In a next step, potential morphing together with electronic structure calculations at the B3LYP and MP2 level of theory was used to align the computed and experimentally observed spectral features in the $\mathrm{OH}$-stretching region. From this analysis, a barrier for double proton transfer between 5 and $7 \mathrm{kcal} \mathrm{mol}^{-1}$ was derived, which compares with a CCSD(T)/ aug-cc-pVTZ calculated barrier of $7.9 \mathrm{kcal} \mathrm{mol}^{-1}$. Such a combination of experimental and computational techniques for estimating barriers for proton transfer in gas phase systems is generic and holds promise for further improved PESs and energetics of these important systems. Additional MD simulations at the semi-empirical DFTB level of theory agree quite well for the center band position but underestimate the width of the $\mathrm{OH}$-stretching band.
\end{abstract}

\section{Introduction}

Aerosols play an important role in atmospheric and health sciences, and organic acids have been investigated as possible precursors to the formation of aerosols. ${ }^{1-5}$ The simplest organic acid, formic acid, is among the most abundant trace gases in the atmosphere, with a concentration on the order of 10 parts per billion by volume detected in urban areas and slightly lower concentrations in rural areas. ${ }^{6-11}$ Formic acid also serves as a

\footnotetext{
${ }^{a}$ Department of Chemistry, University of Copenhagen, Universitetsparken 5 , DK-2100 Copenhagen Ø, Denmark. E-mail: hgk@chem.ku.dk; Fax: +45 35320322; Tel: +4535320334

${ }^{b}$ Department of Chemistry, University of Basel, Klingelbergstrasse 80, 4056 Basel, Switzerland. E-mail: m.meuwly@unibas.ch; Fax: +41 (0)612673855; Tel: +41 (0)612673821

$\dagger$ Electronic supplementary information (ESI) available: Details of the spectral subtraction, parameters for the MMPT force field, point charges used to calculated the dipole moment. Figures of the simulated spectra of FAD and d-FAD with the modified MMPT force fields, a comparison of the potential energy surfaces obtained with the SDM model, SSM model, mixed surface model and at the B3LYP/6-31G(d,p) level of theory, correlation between the MMPT, MP2 and B3LYP energies, and power spectra in the CD-stretching region. See DOI: 10.1039/c6cp03462d
}

useful model system for larger carboxylic acids, especially in theoretical studies, in which calculations on larger organic acids quickly become unfeasible. For this reason, numerous studies on formic acid exist in the literature. ${ }^{12-18}$ In the gas phase, organic acids primarily exist in their dimeric form. These dimers are characterized by a cyclic form and are held together by two hydrogen bonds (see Fig. 1). The ability of the organic acids to form these strong dimers influences their atmospheric impact significantly, as it changes the amount of organic acid available to form precursor complexes with e.g. water.

Infrared spectroscopy is a powerful tool to detect, investigate and characterize hydrogen bonded systems, $\mathrm{XH} \cdots \mathrm{Y}$, where the donor atom $\mathrm{X}$ is more electronegative than $\mathrm{H}$, and $\mathrm{Y}$ is the acceptor atom or group of atoms. ${ }^{19-37}$ The gas-phase infrared spectra of formic acid monomer and dimer have been studied extensively. ${ }^{16,38-49}$ Upon hydrogen bond formation a redshift, intensity enhancement, and line broadening of the XH-stretching transition usually occur. ${ }^{19,20}$ These hydrogen bond characteristics are often used to detect and identify hydrogen bonded systems. However, for formic acid these characteristics complicate the spectrum and its interpretation, as the $\mathrm{OH}$-stretching transition 


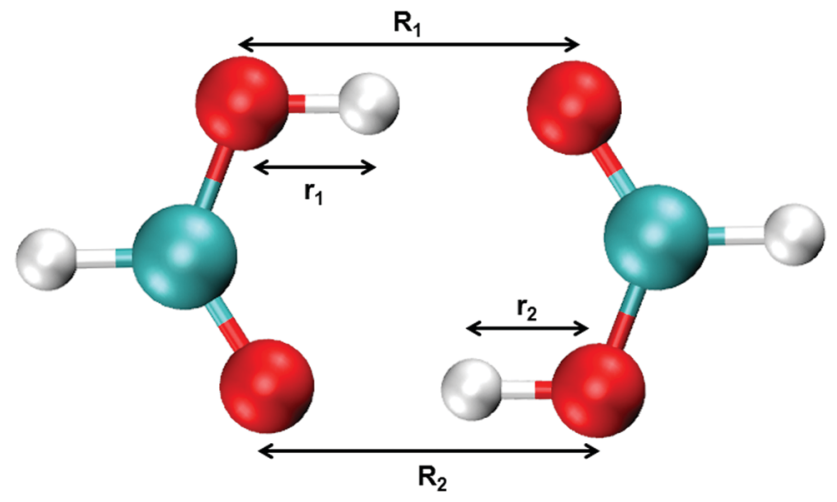

Fig. 1 Structure of formic acid dimer (FAD) with the definition of bond lengths.

shifts and broadens, such that it overlaps with bands in the $\mathrm{CH}$-stretching region. Additionally, the monomer and dimer equilibrium is shifted towards the strongly bound dimer with an equilibrium constant for formic acid dimerization, $K$, of $\sim 300$ at room temperature (values in the range 135-405 have been reported in the literature). ${ }^{13-16,39,50-55}$ As such, features from both the monomer and the dimer will be present at all sample pressures in the gas phase infrared (IR) spectrum, which further complicates band interpretation in the $\mathrm{CH}$ - and $\mathrm{OH}$-stretching region.

Previously, the line shape and broadening of the fundamental $\mathrm{OH}$-stretching band in formic acid dimer were simulated using first-principles anharmonic couplings. ${ }^{45}$ In that study, a cubic force field was calculated along the internal coordinates of the dimer and projected onto a normal mode basis. Mixed vibrational states were generated and the line shape and broadening in the $\mathrm{OH}$-stretching region were predicted, assuming Gaussian line shapes for the calculated vibrational transitions. The experimental broadening and line shape in the $\mathrm{OH}$-stretching region was reproduced reasonably well. The line shape of vibrational transitions depends on several elements such as the transition dipole moment, but it is also related to the dynamics of the system, especially the proton transfer (PT) of formic acid dimer. ${ }^{43}$ Previously, the line shape of the HCl-stretching transition in the $\mathrm{HCl}-$ acetonitrile complex and the $\mathrm{OH}$-stretching transition in the deuterated and undeuterated single proton embedded oxalate anion have been modelled using a combination of ensemble averaging and normal mode harmonic oscillator calculations. ${ }^{56,57}$ In the former, the ensemble averaging is based on an MD simulation, and in the latter, the averaging is based on a Monte Carlo simulation. MD is often used to study dynamic systems and has been used to determine that the two PT motifs in formic acid dimer are coupled. ${ }^{58-60}$ With the advantages of low computing costs, the force field methods have been extensively developed to perform proton transfer reactions in simulations. An early example is the empirical valence bond (EVB) method ${ }^{61}$ which treats a reactive system with resonance forms of ionic and covalent states. The multistate(MS)-EVB method is an extension of EVB which allows simulations with multiple excess protons. ${ }^{62-65}$ More recently, proton transfer reactions have been studied using ReaxFF ${ }^{66}$ and Hydrogen Dynamics, ${ }^{67}$ a method in which a proton moves by morphing between a hydronium ion and a water molecule.

The MMPT force field is more akin to a mixed quantum mechanics/molecular mechanics (QM/MM) formulation. It has been shown to provide QM qualities at much reduced computational cost. ${ }^{68-71}$ Recently, MMPT has been used to study the molecular dynamics and infrared spectrum of the enol form of acetylacetone (AcAc) ${ }^{72}$ The spectral features of AcAc were satisfactorily reproduced and the MD simulations were compared with the measured $\mathrm{OH}$-stretching region to estimate the proton transfer barrier height to be $2.4 \mathrm{kcal} \mathrm{mol}^{-1}$. Furthermore, double proton transfer (DPT) was investigated in a Pt-containing organometallic complex by independent treatment of $\mathrm{NH} \cdots \mathrm{N}$ and $\mathrm{OH} \cdots \mathrm{O}$ as PT motifs, but not including the coupling between the individual PT motifs. ${ }^{73}$

In the present study, we have successfully obtained the isolated infrared spectrum of formic acid dimer (FAD) and the dimer of the deuterated species, DCOOH (d-FAD), in the fundamental $\mathrm{OH}$ - and $\mathrm{CH}$-stretching region by spectral subtraction of a low pressure infrared spectrum of formic acid vapor from a spectrum recorded at a higher pressure. These spectra have been compared with those obtained from MD simulations using a modified MMPT force field generalized to DPT and on-the-fly MD simulations, where the potential energy surface (PES) is calculated using the semi-empirical DFTB method. ${ }^{74-78}$ From the comparison of the experimental and simulated spectra, the barrier height for DPT is estimated.

\section{Methods}

\subsection{Experimental}

Formic acid ( $\mathrm{HCOOH}$, Aldrich, 98\%) and formic-d acid (DCOOH, Aldrich, 98\% D, 95\% in $\mathrm{H}_{2} \mathrm{O}$ ) were purified with several freeze, pump and thaw cycles. The IR spectra were recorded with a VERTEX 70 (Bruker) Fourier transform infrared (FTIR) spectrometer with a $1 \mathrm{~cm}^{-1}$ resolution and 500 scans at room temperature $(296 \pm 1 \mathrm{~K})$. The spectrometer was fitted with a mid-infrared (MIR) light source, $\mathrm{KBr}$ beamsplitter and a liquid nitrogen cooled MCT (Mercury Cadmium Telluride) detector. The spectra of formic acid and formic-d acid were recorded with a $10 \mathrm{~cm}$ and $19 \mathrm{~cm}$ cell, respectively. The samples were introduced in to the cell through a glass vacuum line (J. Young) equipped with valves to control the sample pressure, which was measured with a Varian PCG-750 pressure gauge. The sample was left to equilibrate until the pressure was stable before the spectrum was recorded. The spectral subtractions were performed with OPUS 6.5.

Spectral subtraction. The infrared spectra of FAD and d-FAD were measured in the region from 600 to $4200 \mathrm{~cm}^{-1}$. The equilibrium constant for formic acid dimer formation is large $(\sim 300){ }^{13-16,39,50-55,79}$ and is expected to be of the same order of magnitude for formic-d acid. This is confirmed by calculation of equilibrium constants of complex formation for FA and d-FA at the wB97X-D/aug-cc-pVTZ level of theory using Gaussian09, ${ }^{80}$ as the calculated equilibrium constants for FA and d-FA differ by only $\sim 5 \%$. As a consequence, spectroscopic signatures from 
the dimer are observed even at low sample pressures. The formic acid monomer transitions have a clear rotational structure, and these sharp rotational lines, in combination with a vapor pressure of formic acid that is not sufficiently large to push the equilibrium entirely towards the dimer, mean that features from the monomer are observed even at the highest sample pressures. Consequently, it is difficult to obtain an isolated spectrum of FAD and d-FAD at room temperature, and it has to our knowledge not been reported previously. Careful considerations were necessary in the spectral subtraction in order to obtain the spectrum of the dimer. The spectral subtraction was performed using two spectra, one recorded at high pressure, where the equilibrium is pushed towards the dimer, and the other recorded at a lower pressure, where the equilibrium is pushed towards the monomer. To obtain the spectrum of the dimer, the low-pressure spectrum was scaled and subtracted from the high-pressure spectrum. The low-pressure spectrum was scaled appropriately so that a flat baseline was obtained in the $\mathrm{OH}$-stretching region of the monomer and the rotational lines from the monomer transitions disappear, which indicates that we have obtained the isolated spectrum of pure dimer (see the ESI, $\dagger$ pages S2, S3 and Fig. S1 for details).

\subsection{Molecular dynamics simulations}

MMPT for double proton transfers. Molecular Mechanics with Proton Transfer is a parametrized method to simulate bond breaking and formation between a hydrogen atom (or a proton) and its donor (X) and acceptor (Y), respectively. ${ }^{68-71}$ The total interaction energy for the system with coordinates $\mathbf{Q}$ is:

$$
V(\mathbf{Q})=V_{\mathrm{MM}}(\mathbf{q})+V_{\mathrm{PT}}(R, r, \theta),
$$

where the proton transfer motif $\mathrm{XH}-\mathrm{Y}$ is described by $V_{\mathrm{PT}}(R, r$, $\theta$ ). This contribution is determined from quantum chemical calculations. Here, the coordinates are $R$ (the distance between the $\mathrm{X}$ and $\mathrm{Y}$ atoms), $r$ (the distance between the $\mathrm{X}$ and $\mathrm{H}$ atoms), and $\theta$ (the angle between the unit vectors along $R$ and $r$, see Fig. 1). The dependence of the total potential energy on the remaining degrees of freedom (DOF) of the system $(\mathbf{q})$ is given by a conventional force field $V_{\text {MM }}$. The implementation adds, modifies, and removes force-field terms that include bonded and non-bonded interactions, in a smooth and energy conserving fashion by using switching functions whenever the migrating $\mathrm{H}$ transfers from donor to acceptor. ${ }^{69}$

MMPT treats the proton transfer process in its full dimensionality while addressing three important aspects of the problem: speed, accuracy, and versatility. While speed and accuracy are rooted in the $\mathrm{QM} / \mathrm{MM}$ formulation, the versatility of the approach is exploited by using the morphing potential method. ${ }^{81}$ To this end, it is important to realize that a wide range of proton transfer processes can be described based on three prototype model systems: (a) symmetric single minimum (SSM, the optimized structure of the system has equal sharing of the proton between $\mathrm{X}$ and $\mathrm{Y}$ ), (b) symmetric double-minimum (SDM, the optimized structure of the system has unequal sharing of the proton between $\mathrm{X}$ and $\mathrm{Y}$ but is symmetric with respect to the transition state), and (c) asymmetric single minimum (ASM, the optimized structure of the system has unequal sharing of the proton and is asymmetric with respect to the transition state). ${ }^{69}$ The PES of these three model systems (SSM, SDM, or ASM), can be morphed into a suitable PES to reproduce important topological features of the target PES by a transformation of the type

$$
V_{\operatorname{morph}}\left(R^{\prime}, r^{\prime}, \theta^{\prime}\right)=\lambda(R, r, \theta) V_{\text {orig }}(R, r, \theta),
$$

where $\lambda$ can either be a constant or a more complicated function of one or more coordinates. ${ }^{81}$ In the present work, $\lambda$ was a scalar throughout. The morphing approach not only avoids recomputing a full PES for the PT motif but also reduces the rather laborious task of fitting an entirely new parametrized PES.

Standard MMPT, as described above, treats proton transfers independently and is not necessarily suitable to describe double proton transfer (DPT). ${ }^{73}$ Therefore, a modified MMPT approach is required. Such an extension is afforded by working with a DPT potential, $V_{\mathrm{DPT}}$, which explicitly couples both X-Y distances $\left(R_{1}\right.$, $R_{2}$ ) and both donor-hydrogen distances $\left(r_{1}, r_{2}\right)$ (see Fig. 1 ) in the following fashion:

$$
\begin{aligned}
V_{\mathrm{DPT}} & \left(r_{1}, r_{2}, R_{1}, R_{2}, \theta_{1}, \theta_{2}\right) \\
& =\left[V_{\mathrm{SDM}}\left(r_{1}, R_{1}, \theta_{1}\right) \cdot \gamma+V_{\mathrm{SSM}}\left(r_{1}, R_{1}, \theta_{1}\right) \cdot(1-\gamma)\right] \\
& +\left[V_{\mathrm{SDM}}\left(r_{2}, R_{2}, \theta_{2}\right) \cdot \gamma+V_{\mathrm{SSM}}\left(r_{2}, R_{2}, \theta_{2}\right) \cdot(1-\gamma)\right]
\end{aligned}
$$

where

$$
\begin{aligned}
\gamma\left(r_{1}, r_{2}, R_{1}, R_{2}\right)= & \frac{1}{2}\left\{1+\tanh \left[\sigma \cdot\left(r_{1} \cdot R_{1}-R_{1}^{2} / 2\right)\right]\right. \\
& \left.\cdot \tanh \left[\sigma \cdot\left(r_{2} \cdot R_{2}-R_{2}^{2} / 2\right)\right]\right\}
\end{aligned}
$$

The explicit coupling between the DOFs is in the mixing coefficient, $\gamma\left(r_{1}, r_{2}, R_{1}, R_{2}\right) \in(0,1)$, acting on $V_{\mathrm{SDM}}$ and $V_{\mathrm{SSM}}$. For double proton transfer in FAD, the PES is centrallysymmetric as predicted by the modified MMPT formulation and its QM reference (see Fig. S2, ESI $\dagger$ ). Using standard MMPT with two independent $V_{\text {SDM }}$ potentials for each of the PT motifs yields an unrealistic PES with four global minima. Mixing $V_{\mathrm{SDM}}$ and $V_{\mathrm{SSM}}$ as in the $V_{\mathrm{DPT}}$ potential leads to two isoenergetic minima while eliminating the two other minima, see Fig. S2C (ESI $\dagger$ ). The details of the mixing are determined by $\gamma$ which depends on one free parameter $\sigma$ that can be tuned to reproduce particular reference data.

Force field parametrization. Fitting of $V_{\mathrm{DPT}}$ was carried out with respect to reference calculations at the B3LYP/6-31G(d,p) ${ }^{82,83}$ and MP2/6-311++G(2d,2p $)^{84-87}$ levels of theory. The minimum energy conformation of FAD and the transition state for DPT have been obtained for the two methods and are summarized in Table 1. The corresponding $\operatorname{CCSD}(\mathrm{T}) /$ aug-cc-pVTZ values have been included for comparison. At the MP2 level, the minimum energy conformation of FAD has an O-H bond length of $0.9924 \AA$, slightly longer than that of the formic acid monomer (FAM) of $0.9675 \AA$ due to the hydrogen bonds, and the $\mathrm{O}-\mathrm{O}$ distance is 2.6868 A. B3LYP gives a slightly longer $\mathrm{O}-\mathrm{H}$ bond length and a shorter $\mathrm{O}-\mathrm{O}$ distance. The $\operatorname{CCSD}(\mathrm{T})$ results generally lie in between those of B3LYP and MP2, but closer to the MP2 results. 
Table 1 Selected internal coordinates (in $\AA$ ) of the energy minimum (MIN) and transition state (TS) structure of FAD and its monomer (MON), calculated at the B3LYP/6-31G(d,p) and MP2/6-311++G(2d,2p) level of theory and from their parametrized MMPT force fields. Additionally, the $\mathrm{CCSD}(\mathrm{T})$ /aug-cc-pVTZ calculated internal coordinates are presented for comparison

\begin{tabular}{|c|c|c|c|c|c|}
\hline & \multicolumn{3}{|l|}{ B3LYP } & \multicolumn{2}{|c|}{ MMPT-B3LYP } \\
\hline & MON & MIN & TS & MIN & TS \\
\hline $\mathrm{O}-\mathrm{H}$ & 0.9738 & 1.0075 & 1.2093 & 1.0035 & 1.2089 \\
\hline \multirow[t]{3}{*}{$\mathrm{O}-\mathrm{O}$} & & 2.6509 & 2.4183 & 2.6574 & 2.4178 \\
\hline & \multicolumn{3}{|l|}{ MP2 } & \multicolumn{2}{|c|}{ MMPT-MP2 } \\
\hline & MON & MIN & TS & MIN & TS \\
\hline $\mathrm{O}-\mathrm{H}$ & 0.9675 & 0.9924 & 1.2042 & 0.9871 & 1.2031 \\
\hline \multirow[t]{3}{*}{$\mathrm{O}-\mathrm{O}$} & & 2.6853 & 2.4074 & 2.6868 & 2.4062 \\
\hline & \multicolumn{3}{|c|}{$\operatorname{CCSD}(\mathrm{T})$} & & \\
\hline & MON & MIN & TS & & \\
\hline $\mathrm{O}-\mathrm{H}$ & 0.9701 & 0.9957 & 1.2040 & & \\
\hline $\mathrm{O}-\mathrm{O}$ & & 2.6741 & 2.4070 & & \\
\hline
\end{tabular}

The transition state for DPT in FAD suggests a symmetric conformation with the transferring hydrogens between the two oxygens. Additionally, for the calculated minimum energy conformations of FAD at all the levels of theory, the $\mathrm{OH}-\mathrm{O}$ PT motif is almost but not exactly collinear.

Starting from the minimized structures, PES scans along $R=R_{1}=R_{2}$, ranging from $2.2 \AA$ to $3.2 \AA$ with an increment of $0.1 \AA$ and $r=r_{1}=r_{2}$ from $0.8 \AA$ to $R-0.8 \AA$ with an increment of $0.05 \AA$ A were carried out for both methods in order to parametrize $V_{\text {SDM }}$. For parametrizing $V_{\text {SSM }}$ similar PES scans were carried out for $r_{1}=R-r_{2}$. Next, the parameter $\sigma$ in eqn (4) has been fitted to best reproduce the target data at the B3LYP level, which yields $\sigma=2.639 \AA^{-2}$. The quality of this fit is $r^{2}=0.999$ for B3LYP as the target energies, and $r^{2}=0.998$ for MP2. Given this good agreement, no further optimization of $\sigma$ was considered for the MP2 reference data. The two PESs are labelled as MMPT-B3LYP and MMPT-MP2 in the following, respectively (see Fig. S3, ESI $\dagger$ ).

The fitted MMPT-MP2 PES has an optimized $\mathrm{O}-\mathrm{H}$ bond length of $0.9871 \AA$ which differs by $\sim 0.005 \AA$ from the MP2 reference calculations, and the $\mathrm{O}-\mathrm{O}$ distance differs even less. On the other hand, B3LYP gives a slightly longer $\mathrm{O}-\mathrm{H}$ bond length and a shorter $\mathrm{O}-\mathrm{O}$ distance. For the TS structures, both MMPT-MP2 and MMPT-B3LYP yield results close to their respective QM references. Moreover, the reaction barriers were well reproduced to within $<0.1 \mathrm{kcal} \mathrm{mol}^{-1}$, which further establishes the quality of the parametrized PESs.

Simulation details. All NVE MD simulations were performed with CHARMM ${ }^{88-90}$ in the gas phase at $300 \mathrm{~K}$, following heating and equilibration ( $1 \mathrm{ps}$ for each trajectory with $\Delta t=0.1 \mathrm{fs}$ ). Since DPT involves an appreciable barrier $\left(7.9 \mathrm{kcal} \mathrm{mol}^{-1}\right.$ at the $\operatorname{CCSD}(\mathrm{T})$ level), $100 \mathrm{~ns}$ production runs have been carried out. For direct comparison, on-the-fly molecular dynamics at the semi-empirical DFTB ${ }^{77,91,92}$ level (MD-DFTB) have been carried out for $10 \mathrm{~ns}$ with the same simulation conditions as for the force field simulations described above.
Infrared spectra. The infrared spectrum is computed from the total dipole moment, $\vec{M}(t)$, obtained from each step in the MD trajectory. The dipole-dipole correlation, $C(t)$, is given by

$$
C(t)=\langle\vec{M}(0) \vec{M}(t)\rangle
$$

where $\vec{M}(t)$ is the total molecular dipole moment at time $t$ along the MD trajectory determined from the charge model given in Table S3 (ESI $\dagger$ ). The angular brackets denote an average over the time origins. If $C(\omega)$, the Fourier transform of $C(t)$, is weighted with the Boltzmann distribution, the classical infrared spectrum, $A(\omega)$, is obtained: ${ }^{93,94}$

$$
A(\omega)=\omega\left\{1-\exp \left[-\hbar \omega /\left(k_{\mathrm{B}} T\right)\right]\right\} C(\omega)
$$

where $\omega$ is the transition frequency, $k_{\mathrm{B}}$ is the Boltzmann constant and $T$ is the temperature. Such an approach does not satisfy detailed balance $I(\omega)=\exp \left(h \omega / k_{\mathrm{B}} T\right) I(-\omega)$ because it is derived from a classical correlation function for which $I_{\mathrm{cl}}(\omega)=$ $I_{\mathrm{cl}}(-\omega) .{ }^{95}$ To remedy this, various quantum correction factors (qcf) have been proposed. ${ }^{95,96}$ On the other hand, it has been found that different qcf yield results of different quality for formaldehyde. ${ }^{97}$ Hence it is not a priori clear which of the qcf to choose for a particular problem. Furthermore, in the classical limit $\lim _{T \rightarrow \infty} \exp \left(\hbar \omega / k_{\mathrm{B}} T\right)=1$. Therefore, the spectra reported here are all determined from eqn (6). It is also possible to determine power spectra corresponding to specific internal coordinates, $q$, from the MD trajectory. This is particularly useful to assign spectroscopic features to the motion along these coordinates and identify couplings between internal degrees of freedom. For this purpose, the correlation function $\langle q(0) q(t)\rangle$ is Fourier transformed and weighted with the Boltzmann distribution to yield the power spectrum.

\section{Results and discussion}

We have obtained isolated gas phase spectra of the pure FAD and pure d-FAD at room temperature as described in the Experimental section, and show these in Fig. 2. The spectral signatures for the two systems are very similar, especially the structure of the band associated with the $\mathrm{OH}$-stretching transition. As expected upon deuteration, the fundamental $\mathrm{CH} / \mathrm{CD}$-stretching transition is shifted from $2939 \mathrm{~cm}^{-1}$ in the FAD spectrum to $2210 \mathrm{~cm}^{-1}$ in the spectrum of d-FAD. The OH-stretching transition in both spectra is very broad and the transition assigned to the $\mathrm{OH}$-stretch is observed in the region from $2600-3400 \mathrm{~cm}^{-1}$ in agreement with previously recorded spectra that contain a mixture of formic acid monomer and dimer. ${ }^{40,48}$ Broad $\mathrm{OH}$-stretching transitions are very common in hydrogen bonded complexes ${ }^{72,98,99}$ and have also been observed for overtone transitions of the intramolecular hydrogen bond in pyruvic acid. ${ }^{100}$ It is the spectral features in the $\mathrm{OH}$-stretching region that are of particular interest here as these features are ideally suited to relate experiment, proton transfer energetics and dynamics, and computed IR spectra. Typically, such spectral features are not readily explained by standard static vibrational 


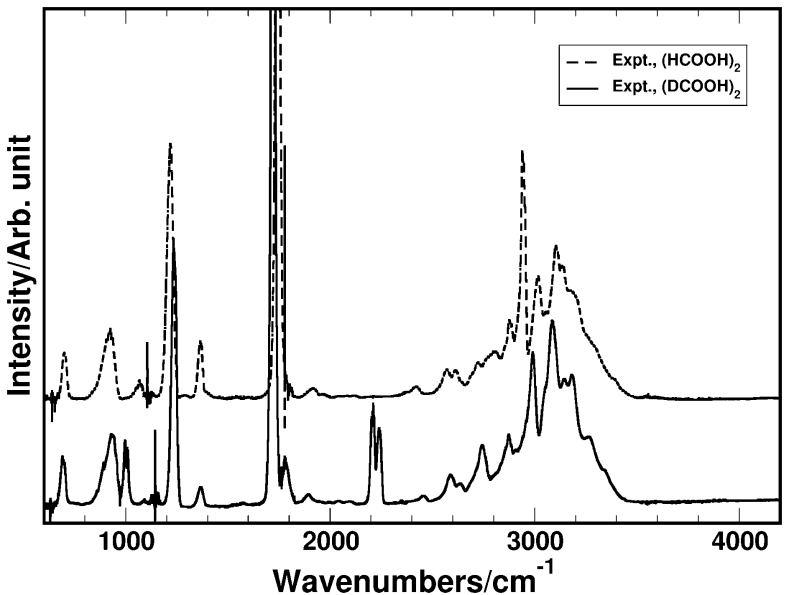

Fig. 2 Experimentally recorded IR spectra of FAD (upper dashed trace) and d-FAD (lower solid trace).

models such as the harmonic oscillator normal mode model, anharmonic local mode theory, or second-order vibrational perturbation theory (VPT2). ${ }^{101-103}$

The MD simulated IR-spectra of FAD with the different PESs are compared in Fig. 3. In the region near $3000 \mathrm{~cm}^{-1}$, spectral features arise from a very broad $\mathrm{OH}$-stretching band and a sharp CH-stretching peak near $2900 \mathrm{~cm}^{-1}$. The $\mathrm{OH}-$ and $\mathrm{CH}$-stretching power spectra are compared with the measured IR-spectrum in Fig. 3. The position of the $\mathrm{CH}$-stretching peak predicted by the MD simulated spectra compares well with that of the experimental spectrum. However, the broad $\mathrm{OH}$-stretching band is blue shifted compared to that of the experiment in all three simulations in Fig. 3. Recently, it was shown that the center frequency of the broad $\mathrm{OH}$-stretching band in acetylacetone was affected significantly by the height of the proton transfer barrier. ${ }^{72}$ This is not surprising, as a change in the barrier height inevitably affects the shape of the PES in the region near the two global minima. In order to investigate the correlation between the OH-stretching peak position and the DPT reaction barrier, the height of the reaction barrier was changed in the MMPT-MP2 force field by morphing with the parameter $\lambda$ (see the Methods section). The MMPT-MP2 force field has a DPT barrier height of $8.2 \mathrm{kcal} \mathrm{mol}^{-1}$ and features from the OH-stretching transition were observed centered around $3300 \mathrm{~cm}^{-1}$. If the barrier height is lowered to, for example, 5.2 or $2.2 \mathrm{kcal} \mathrm{mol}^{-1}$ the center of the $\mathrm{OH}$-stretching transition red shifts significantly to $\sim 2700$ or $\sim 1700 \mathrm{~cm}^{-1}$, respectively, compared to the original spectrum (see Fig. 4). All remaining transitions are more or less unaffected.

The unmorphed barrier height of the MMPT-B3LYP force field is $5.4 \mathrm{kcal} \mathrm{mol}^{-1}$, which is similar to the morphed MMPTMP2 barrier height $\left(5.2 \mathrm{kcal} \mathrm{mol}^{-1}\right)$. However, the centers of the $\mathrm{OH}$-stretching bands in the two infrared spectra differ substantially (see Fig. 3 and 4), despite the fact that the barrier heights only differ by $0.2 \mathrm{kcal} \mathrm{mol}^{-1}$. Hence, factors other than the barrier height alone, such as the local curvature of the PES, influence the position of the $\mathrm{OH}$-stretching band position. We found that if the proton transfer reaction barriers are morphed to $5.1 \mathrm{kcal} \mathrm{mol}^{-1}$

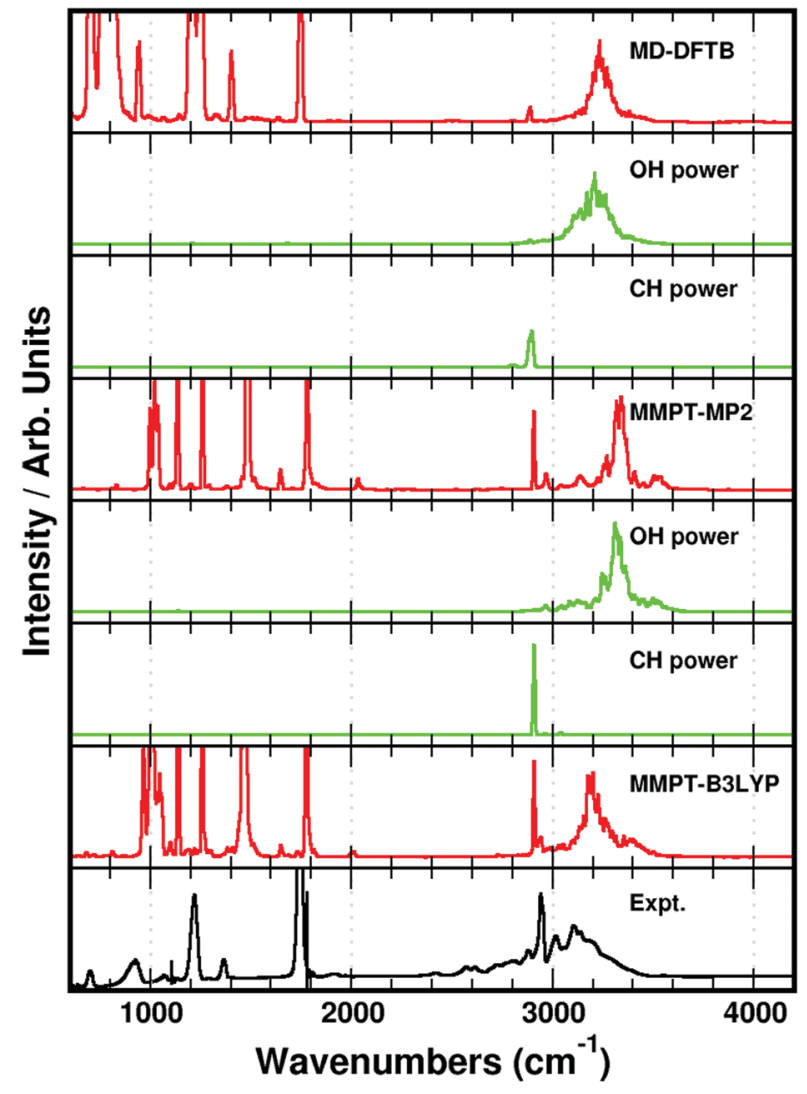

Fig. 3 Spectra obtained from MD simulations with different energy functions. From top to bottom: MD-DFTB (DPT barrier of $7.5 \mathrm{kcal} \mathrm{mol}^{-1}$ ) and its $\mathrm{OH}$ and $\mathrm{CH}$ power spectra (green); MMPT-MP2 (DPT barrier of $8.2 \mathrm{kcal} \mathrm{mol}^{-1}$ ) and the $\mathrm{OH}$ and $\mathrm{CH}$ power spectra (green), and MMPTB3LYP (DPT barrier of $5.4 \mathrm{kcal} \mathrm{mol}^{-1}$ ). The experimentally recorded spectrum of FAD has been included for comparison (bottom).

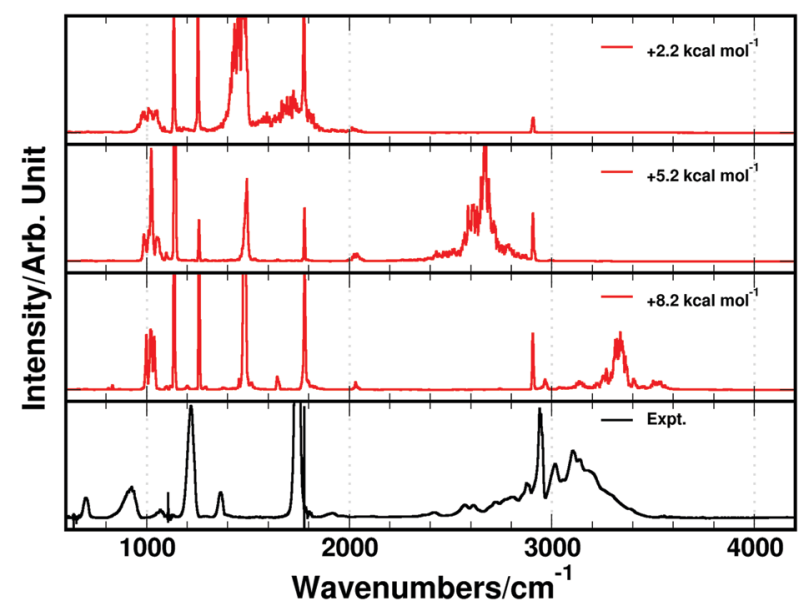

Fig. 4 MD simulated spectra with the MMPT-MP2 force field of FAD, with morphed barrier heights of $2.2 \mathrm{kcal} \mathrm{mol}^{-1}$ (top), $5.2 \mathrm{kcal} \mathrm{mol}^{-1}$ (middle) and $8.2 \mathrm{kcal} \mathrm{mol}^{-1}$ (unscaled, bottom). The experimental spectrum has been included for comparison.

and $7.2 \mathrm{kcal} \mathrm{mol}^{-1}$ for the MMPT-B3LYP and MMPT-MP2 force field, respectively, the position of the simulated $\mathrm{OH}$-stretching band fits well with the experimental $\mathrm{OH}$-stretching band position. 
In other words, the barrier to proton transfer can at best be determined to within $\pm 1 \mathrm{kcal} \mathrm{mol}^{-1}$. In comparison, the CCSD(T)/aug-cc-pVTZ calculated barrier is $7.9 \mathrm{kcal} \mathrm{mol}^{-1}$ using MOLPRO, ${ }^{104}$ which is close to the barrier of $7.2 \mathrm{kcal} \mathrm{mol}^{-1}$ found with the morphed MMPT-MP2 force field, but is higher than the barrier obtained with the MMPT-B3LYP force field.

Up to this point the parameters in the conventional force field (see $V_{\mathrm{MM}}(\mathbf{q})$ in eqn (1)) were those of the C36 force field ${ }^{105}$ and the only modifications of the MMPT-force field concerned the height of the barrier for DPT. Considering Fig. 3 and 4 slight adjustments in the force field may improve in particular the position of the $\mathrm{CH}$-stretching band. Hence, in order to improve the simulated spectra the harmonic force constant for the $\mathrm{CH} / \mathrm{CD}$-stretch was rescaled from 330 to $340 \mathrm{kcal} \mathrm{mol}^{-1} \AA^{-2}$. Furthermore, $a b$ initio calculations at the B3LYP/6-31G(d,p) level along the bending potential of the transferring hydrogen (HOObend) in FAD suggest that the bending force constant from the SSM and SDM model potentials $\left(k=33 \mathrm{kcal} \mathrm{mol}^{-1} \mathrm{rad}^{-2}\right.$, see eqn (S3) in ESI $\dagger$ ) should be decreased to $k=17.5 \mathrm{kcal} \mathrm{mol}^{-1} \mathrm{rad}^{-2}$ to match the reference calculations. With these adjustments the IR spectra for FAD and d-FAD were recomputed and are reported in Fig. 5. For the simulated spectra with the modified DPT barriers and force constants the agreement in the fundamental $\mathrm{OH}$-stretching region compared to that of the experimental spectrum has improved (see Fig. 5). For the B3LYP-MMPT force field, the $\mathrm{CH}$-stretching frequency for FAD shifts from 2910 to $2950 \mathrm{~cm}^{-1}$ as the arrow around $2900 \mathrm{~cm}^{-1}$ in the left hand panel of Fig. 5 indicates. Also, the $\mathrm{H}$-transfer band shifts its maximum from $3200 \mathrm{~cm}^{-1}$ to $3100 \mathrm{~cm}^{-1}$ upon morphing the barrier height from 5.4 to $5.1 \mathrm{kcal} \mathrm{mol}{ }^{-1}$. The signal at $\sim 2700 \mathrm{~cm}^{-1}$
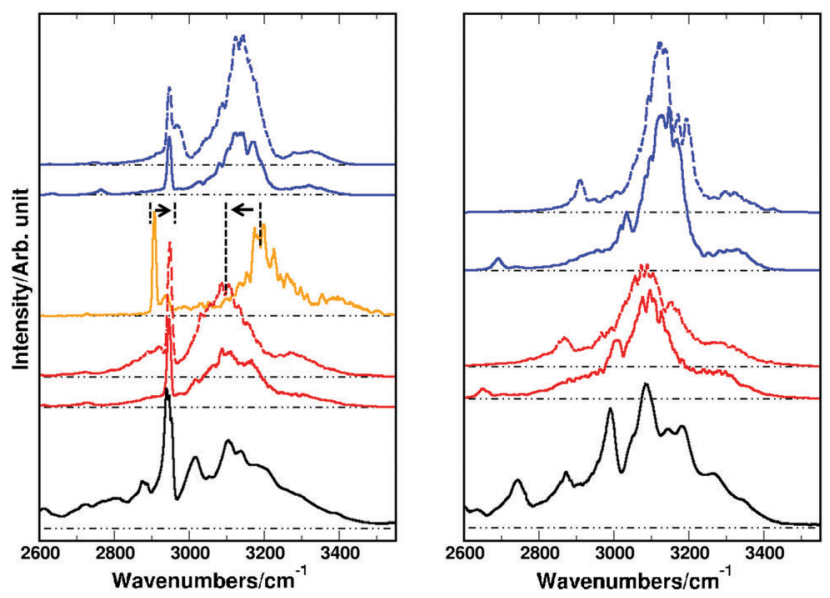

Fig. 5 Comparison of experimental spectra (black traces) with simulated spectra (average over 10 independent MD simulations of 100 ns each) for FAD (left panel) and d-FAD (right panel). Red and blue traces for the MMPTB3LYP and MMPT-MP2 parametrizations, respectively, with morphed barriers of $5.1 \mathrm{kcal} \mathrm{mol}^{-1}$ and $7.2 \mathrm{kcal} \mathrm{mol}^{-1}$ and the $\mathrm{CH}$-stretching force constant adjusted to reproduce the experimentally observed value. Solid traces for simulations with $k=33 \mathrm{kcal} \mathrm{mol}^{-1} \mathrm{rad}^{-2}$ and dashed traces for $k=17.5 \mathrm{kcal} \mathrm{mol}^{-1} \mathrm{rad}^{-2}$ (see text). The orange line in the left panel is for the unmorphed DPT barrier height (MMPT-B3LYP) and the original $\mathrm{CH}$ force constant (330 kcal mol $\mathrm{mo}^{-1} \AA^{-2}$ ) with the arrows indicating in which direction the bands shift upon changing the force field. for d-FAD with $k=33 \mathrm{kcal} \mathrm{mol}^{-1} \mathrm{rad}^{-2}$ shifts to $2900 \mathrm{~cm}^{-1}$ for $k=17.5 \mathrm{kcal} \mathrm{mol}^{-1} \mathrm{rad}^{-2}$ and can be associated with the unbound $\mathrm{H}-\mathrm{O}$ stretch frequency from analysis of the power spectra, however, the mode is heavily mixed. The width and position of the $\mathrm{OH}^{-}$and $\mathrm{CH}$-stretching band depend only little on the bending force constant, $k$, (see dashed and solid lines in Fig. 5) and are satisfactorily reproduced compared to previous line shape studies on formic and acetic acid dimer. ${ }^{45,106}$ Depending on the force constant $k$, the fundamental $\mathrm{COH}$-bend is located at $\sim 1400 \mathrm{~cm}^{-1}$ and $\sim 1500 \mathrm{~cm}^{-1}$ for $k=17.5$ and $k=33 \mathrm{kcal} \mathrm{mol}^{-1} \mathrm{rad}^{-2}$ for FAD, respectively. Experimentally this transition is observed at $1220 \mathrm{~cm}^{-1}$.

It is also of interest to comment on the clearly discernible and almost equally-spaced progressions $\left(\sim 125 \mathrm{~cm}^{-1}\right)$ in the experimental spectra. Most of the sub-structure in the $\mathrm{OH}$-stretching region have previously been attributed the coupling between the $\mathrm{OH}$-stretch and the symmetric and anti-symmetric $\mathrm{COH}$-bend. ${ }^{45}$ Giese et al. observed a similar progression in the $\mathrm{OH}$-stretching region of the simulated stick spectrum of malonaldehyde. ${ }^{107}$ They found that the progression was a result of strong mixing of the intramolecular hydrogen bound $\mathrm{OH}$-stretching vibration with the in-plane $\mathrm{OH}$-bending vibration. As such, we conclude that the progressions in the $\mathrm{OH}$-stretching spectra of FAD and $\mathrm{d}-\mathrm{FAD}$ is due to the coupling between the $\mathrm{OH}$-stretching vibration and vibrations that partially break the hydrogen bond such as the $\mathrm{COH}$-bending and in-plane $\mathrm{OH}$-bending vibration.

During 250 ns of MD simulations with the MMPT-B3LYP (with the morphed barrier height of $5.1 \mathrm{kcal} \mathrm{mol}^{-1}$ ) 25 proton transfers were observed, i.e. corresponding to a DPT rate of $0.1 \mathrm{~ns}^{-1}$. Test calculations show that irrespective of the number of DPT events the IR spectra are all very similar. For this, IR spectra were determined over several separate $50 \mathrm{~ns}$ intervals and compared. In the $250 \mathrm{~ns}$ simulations with the MMPT-MP2 PES (with the unmorphed barrier of $8.2 \mathrm{kcal} \mathrm{mol}^{-1}$ ) and in the 10 ns MD-DFTB simulation no DPT was observed. Previously, PT has been explicitly been linked to the spectra features of the $\mathrm{OH}$-stretching band, ${ }^{43}$ but we find here that the actual occurrence of DPT is not necessarily required for observing a broad $\mathrm{OH}$-absorption in the IR spectrum.

One increasingly important aspect of current force field development is the issue of transferability, i.e. the question how easily a given parametrization can be adapted to a chemically related situation. For halogenated molecules this has recently been assessed and it was found that scaling van der Waals parameters can lead to largely transferable parametrizations. ${ }^{108}$ For singleand double proton transfer the overall shape (symmetric single minimum, symmetric double minimum, asymmetric double minimum) of the potential energy surface is usually known $a$ priori. Hence, starting from a correct topology, morphing transformations between the correct topology and the target PES, characterized by the barrier height and the relative stabilizations of the two minima (for asymmetric double minimum), morphing transformations will be an efficient means to develop appropriate force fields for a new system. As an example, for derivatives $\mathrm{RCOOH}-\mathrm{HOOCR}$ of $\mathrm{FAD}$ (e.g. acetic acid dimer) it is reasonable to assume that the general topology of the PES is related to that of FAD. 
Depending on the chemical identity of the R-group (e.g. $\mathrm{CH}_{3}$, halogen) the height of the DPT barrier will change, though. This can be accounted for by suitable linear or nonlinear morphing transformations. Hence, with a limited number of electronic structure calculations the necessary information about the target PES can be obtained and the morphing parameters which describe DPT in RCOOH-HOOCR can be determined.

\section{Conclusion}

The vibrational spectroscopy of FAD has been investigated experimentally and by computer simulations. Spectra at low pressures of formic acid, $\mathrm{HCOOH}$, and formic-d acid, $\mathrm{DCOOH}$, vapor were recorded to push the equilibrium towards the monomer. These spectra were scaled and subtracted from the corresponding spectra recorded at higher pressures of formic acid and formic-d acid vapor, where the equilibrium is pushed towards the dimer. Successful subtractions of the monomer from the high pressure spectra were achieved, and the isolated gas phase spectra of formic acid and formic-d acid dimer (FAD and d-FAD) at room temperature were obtained. A modified version of the MMPT force field was developed and successfully applied to FAD and d-FAD to facilitate interpretation of the isolated dimer spectra in the $\mathrm{OH}$-stretching region. After rescaling the proton transfer barrier of the MMPT force field and changing the $\mathrm{CH} / \mathrm{CD}$-stretching and HOO-bending force constants, the computed infrared spectra of FAD and d-FAD favorably agreed with those from experiment in the $\mathrm{OH}$-stretching region. The scaled proton transfer barrier of $7.2 \mathrm{kcal} \mathrm{mol}^{-1}$ in the MMPTMP2 force field was found to be comparable to the barrier height of $7.9 \mathrm{kcal} \mathrm{mol}^{-1}$ obtained at the $\operatorname{CCSD}(\mathrm{T}) /$ aug-cc-pVTZ level of theory. However, the scaled barrier height includes entropic contributions as it involves dynamics on the fulldimensional PES from which the spectroscopy was determined. The $T=0 \mathrm{~K}$ calculation at the higher $\operatorname{CCSD}(\mathrm{T}) /$ aug-cc-PVTZ level of theory does not include such entropic contributions. It is reassuring that the $T=0 \mathrm{~K}$ value is an upper bound to the barrier height from finite-temperature MD simulations, as it should be. The scaled barrier height in the MMPT-B3LYP force field that provided a good fit of the central $\mathrm{OH}$-stretching frequency was lower by $2 \mathrm{kcal} \mathrm{mol}^{-1}$ compared to that of the MMPT-MP2 force field which is considered to be the more realistic value. The present work shows that MMPT can be extended to treat DPT in a meaningful fashion which is also applicable to larger carboxylic acids where more computationally demanding on-the-fly MD simulations are not feasible.

\section{Acknowledgements}

We thank the Danish Council for Independent Research-Natural Sciences, the Danish Center for Scientific Computing (DCSC), and the Department of Chemistry at the University of Copenhagen for funding. KM is grateful to the Ministry of Higher Education and Science for the "EliteForsk" travel scholarship. The work in Basel is supported by the Swiss National Science
Foundation (Grant 200020-132406 and the NCCR MUST) and the University of Basel.

\section{References}

1 G. Myhre, D. Shindell, F.-W. Bréon, W. Collins, J. Fuglestvedt, J. Huang, D. Koch, J.-F. Lamarque, D. Lee, B. Mendoza, T. Nakajima, A. Robock, G. Stephens, T. Takamura and H. Zhang, Climate Change 2013: The Physical Science Basis. Contribution of Working Group I to the Fifth Assessment Report of Intergovernmental Panel on Climate Change, 2013.

2 A. Chebbi and P. Carlier, Atmos. Environ., 1996, 30, 4233-4249. 3 S. Yu, Atmos. Res., 2000, 53, 185-217.

4 M. Kanakidou, J. H. Seinfeld, S. N. Pandis, I. Barnes, F. J. Dentener, M. C. Facchini, R. Van Dingenen, B. Ervens, A. Nenes, C. J. Nielsen, E. Swietlicki, J. P. Putaud, Y. Balkanski, S. Fuzzi, J. Horth, G. K. Moortgat, R. Winterhalter, C. E. L. Myhre, K. Tsigaridis, E. Vignati, E. G. Stephanou and J. Wilson, Atmos. Chem. Phys., 2005, 5, 1053-1123.

5 M. Hallquist, J. C. Wenger, U. Baltensperger, Y. Rudich, D. Simpson, M. Claeys, J. Dommen, N. M. Donahue, C. George, A. H. Goldstein, J. F. Hamilton, H. Herrmann, T. Hoffmann, Y. Iinuma, M. Jang, M. E. Jenkin, J. L. Jimenez, A. Kiendler-Scharr, W. Maenhaut, G. McFiggans, T. F. Mentel, A. Monod, A. S. H. Prévôt, J. H. Seinfeld, J. D. Surratt, R. Szmigielski and J. Wildt, Atmos. Chem. Phys., 2009, 9, 5155-5236.

6 G. A. Dawson, J. C. Farmer and J. L. Moyers, Geophys. Res. Lett., 1980, 7, 725-728.

7 J. C. Farmer and G. A. Dawson, J. Geophys. Res.: Oceans, 1982, 87, 8931-8942.

8 P. L. Hanst, N. W. Wong and J. Bragin, Atmos. Environ., 1982, 16, 969-981.

9 R. W. Talbot, K. M. Beecher, R. C. Harriss and W. R. Cofer, J. Geophys. Res.: Atmos., 1988, 93, 1638-1652.

10 M. O. Andreae, R. W. Talbot, T. W. Andreae and R. C. Harriss, J. Geophys. Res.: Atmos., 1988, 93, 1616-1624.

11 J. E. Lawrence and P. Koutrakis, Environ. Sci. Technol., 1994, 28, 957-964.

12 L. George and W. Sander, Spectrochim. Acta, Part A, 2004, 60, 3225-3232.

13 M. D. Taylor and J. Bruton, J. Am. Chem. Soc., 1952, 74, 4151-4152.

14 J. R. Barton and C. C. Hsu, J. Chem. Eng. Data, 1969, 14, 184-187.

15 R. Büttner and G. Maurer, Ber. Bunsenges. Phys. Chem., 1983, 87, 877-882.

16 J. Vander Auwera, K. Didriche, A. Perrin and F. Keller, J. Chem. Phys., 2007, 126, 124311.

17 J. E. Bertie and K. H. Michaelian, J. Chem. Phys., 1982, 76, 886-894.

18 J. E. Bertie, K. H. Michaelian, H. H. Eysel and D. Hager, J. Chem. Phys., 1986, 85, 4779-4789.

19 E. Arunan, G. R. Desiraju, R. A. Klein, J. Sadlej, S. Scheiner, I. Alkorta, D. C. Clary, R. H. Crabtree, J. J. Dannenberg, 
P. Hobza, H. G. Kjaergaard, A. C. Legon, B. Mennucci and D. J. Nesbitt, Pure Appl. Chem., 2011, 83, 1619-1636.

20 E. Arunan, G. R. Desiraju, R. A. Klein, J. Sadlej, S. Scheiner, I. Alkorta, D. C. Clary, R. H. Crabtree, J. J. Dannenberg, P. Hobza, H. G. Kjaergaard, A. C. Legon, B. Mennucci and D. J. Nesbitt, Pure Appl. Chem., 2011, 83, 1637-1641.

21 S. Chung and M. Hippler, J. Chem. Phys., 2006, 124, 214316. 22 M. Hippler, J. Chem. Phys., 2007, 127, 084306.

23 M. Hippler, S. Hesse and M. A. Suhm, Phys. Chem. Chem. Phys., 2010, 12, 13555-13565.

24 B. Michielsen, J. J. J. Dom, B. J. v. d. Veken, S. Hesse, Z. Xue, M. A. Suhm and W. A. Herrebout, Phys. Chem. Chem. Phys., 2010, 12, 14034-14044.

25 L. Du and H. G. Kjaergaard, J. Phys. Chem. A, 2011, 115, 12097-12104.

26 J. J. J. Dom, B. J. van der Veken, B. Michielsen, S. Jacobs, Z. Xue, S. Hesse, H.-M. Loritz, M. A. Suhm and W. A. Herrebout, Phys. Chem. Chem. Phys., 2011, 13, 14142-14152.

27 L. Du, J. R. Lane and H. G. Kjaergaard, J. Chem. Phys., 2012, 136, 184305.

28 B. Michielsen, C. Verlackt, B. van der Veken and W. Herrebout, J. Mol. Struct., 2012, 1023, 90-95.

29 L. Du, K. Mackeprang and H. G. Kjaergaard, Phys. Chem. Chem. Phys., 2013, 15, 10194-10206.

30 N. Bork, L. Du and H. G. Kjaergaard, J. Phys. Chem. A, 2014, 118, 1384-1389.

31 N. Bork, L. Du, H. Reiman, T. Kurtèn and H. G. Kjaergaard, J. Phys. Chem. A, 2014, 118, 5316-5322.

32 A. S. Hansen, L. Du and H. G. Kjaergaard, Phys. Chem. Chem. Phys., 2014, 16, 22882-22891.

33 A. S. Hansen, L. Du and H. G. Kjaergaard, J. Phys. Chem. Lett., 2014, 5, 4225-4231.

34 C. L. Andersen, C. S. Jensen, K. Mackeprang, L. Du, S. Jørgensen and H. G. Kjaergaard, J. Phys. Chem. A, 2014, 118, 11074-11082.

35 K. H. Møller, A. S. Hansen and H. G. Kjaergaard, J. Phys. Chem. A, 2015, 119, 10988-10998.

36 M. Heger, M. A. Suhm and R. A. Mata, J. Chem. Phys., 2014, 141, 101105.

37 M. Heger, R. A. Mata and M. A. Suhm, Chem. Sci., 2015, 6, 3738-3745.

38 L. G. Bonner and R. Hofstadter, J. Chem. Phys., 1938, 6, 531-534.

39 A. Clague and H. Bernstein, Spectrochim. Acta, Part A, 1969, 25, 593-596.

40 P. Excoffon and Y. Maréchal, Spectrochim. Acta, Part A, 1972, 28, 269-283.

41 Y. Maréchal, J. Chem. Phys., 1987, 87, 6344-6353.

42 D. Hurtmans, F. Herregodts, M. Herman, J. Liévin, A. Campargue, A. Garnache and A. A. Kachanov, J. Chem. Phys., 2000, 113, 1535-1545.

43 M. V. Vener, O. Kühn and J. M. Bowman, Chem. Phys. Lett., 2001, 349, 562-570.

44 M. Freytes, D. Hurtmans, S. Kassi, J. Liévin, J. V. Auwera, A. Campargue and M. Herman, Chem. Phys., 2002, 283, 47-61.
45 G. M. Florio, T. S. Zwier, E. M. Myshakin, K. D. Jordan and E. L. Sibert, J. Chem. Phys., 2003, 118, 1735-1746.

46 R. Georges, M. Freytes, D. Hurtmans, I. Kleiner, J. V. Auwera and M. Herman, Chem. Phys., 2004, 305, 187-196.

47 D. L. Howard and H. G. Kjaergaard, J. Chem. Phys., 2004, 121, 136-140.

48 F. Ito, J. Chem. Phys., 2008, 128, 114310.

49 A. Perrin, J. V. Auwera and Z. Zelinger, J. Quant. Spectrosc. Radiat. Transfer, 2009, 110, 743-755.

50 H. C. Ramsperger and C. W. Porter, J. Am. Chem. Soc., 1926, 48, 1267-1273.

51 A. S. Coolidge, J. Am. Chem. Soc., 1928, 50, 2166-2178.

52 R. C. Herman, J. Chem. Phys., 1940, 8, 252-258.

53 A. Winkler and P. Hess, J. Am. Chem. Soc., 1994, 116, 9233-9240.

54 S. Miyamoto, S. Nakamura, Y. Iwai and Y. Arai, J. Chem. Eng. Data, 1999, 44, 48-51.

55 F. Kollipost, R. W. Larsen, A. V. Domanskaya, M. Nörenberg and M. A. Suhm, J. Chem. Phys., 2012, 136, 151101.

56 N. Bork, V. Loukonen, H. G. Kjaergaard and H. Vehkamaki, Phys. Chem. Chem. Phys., 2014, 16, 24685-24690.

57 C. T. Wolke, A. F. DeBlase, C. M. Leavitt, A. B. McCoy and M. A. Johnson, J. Phys. Chem. A, 2015, 119, 13018-13024.

58 H. Ushiyama and K. Takatsuka, J. Chem. Phys., 2001, 115, 5903-5912.

59 P. R. L. Markwick, N. L. Doltsinis and D. Marx, J. Chem. Phys., 2005, 122, 054112.

60 L. C. T. Pierce, P. R. L. Markwick, J. A. McCammon and N. L. Doltsinis, J. Chem. Phys., 2011, 134, 174107.

61 A. Warshel and R. M. Weiss, J. Am. Chem. Soc., 1980, 102, 6218-6226.

62 U. Schmitt and G. Voth, J. Phys. Chem. B, 1998, 102, 5547-5551.

63 T. Day, A. Soudackov, M. Cuma, U. Schmitt and G. Voth, J. Chem. Phys., 2002, 117, 5839-5849.

64 Y. Wu, H. Chen, F. Wang, F. Paesani and G. A. Voth, J. Phys. Chem. B, 2008, 112, 467-482.

65 J. M. J. Swanson, C. M. Maupin, H. Chen, M. K. Petersen, J. Xu, Y. Wu and G. A. Voth, J. Phys. Chem. B, 2007, 111, 4300-4314.

66 O. Rahaman, A. C. T. van Duin, W. A. Goddard, III and D. J. Doren, J. Phys. Chem. B, 2011, 115, 249-261.

67 M. G. Wolf and G. Groenhof, J. Comput. Chem., 2014, 35, 657-671.

68 S. Lammers and M. Meuwly, J. Phys. Chem. A, 2007, 111, 1638-1647.

69 S. Lammers, S. Lutz and M. Meuwly, J. Comput. Chem., 2008, 29, 1048-1063.

70 Y. Yang and M. Meuwly, J. Chem. Phys., 2010, 133, 064503.

71 S. Lutz, I. Tubert-Brohman, Y. Yang and M. Meuwly, J. Biol. Chem., 2011, 286, 23679-23687.

72 D. L. Howard, H. G. Kjaergaard, J. Huang and M. Meuwly, J. Phys. Chem. A, 2015, 119, 7980-7990.

73 J. Huang, D. Haeussinger, U. Gellrich, W. Seiche, B. Breit and M. Meuwly, J. Phys. Chem. B, 2012, 116, 14406-14415.

74 M. Elstner, D. Porezag, G. Jungnickel, J. Elsner, M. Haugk, T. Frauenheim, S. Suhai and G. Seifert, Phys. Rev. B: Condens. Matter Mater. Phys., 1998, 58, 7260-7268. 
75 M. Elstner, T. Frauenheim, E. Kaxiras, G. Seifert and S. Suhai, Phys. Status Solidi B, 2000, 217, 357-376.

76 T. Frauenheim, G. Seifert, M. Elstner, T. Niehaus, C. Köhler, M. Amkreutz, M. Sternberg, Z. Hajnal, A. D. Carlo and S. Suhai, J. Phys.: Condens. Matter, 2002, 14, 3015.

77 Q. Cui, M. Elstner, E. Kaxiras, T. Frauenheim and M. Karplus, J. Phys. Chem. B, 2001, 105, 569-585.

78 J. Pu, J. Gao and D. G. Truhlar, J. Phys. Chem. A, 2004, 108, 5454-5463.

79 Z. Maroun, K. Mackeprang and H. G. Kjaergaard, 2016, unpublished work.

80 M. J. Frisch, G. W. Trucks, H. B. Schlegel, G. E. Scuseria, M. A. Robb, J. R. Cheeseman, G. Scalmani, V. Barone, B. Mennucci, G. A. Petersson, H. Nakatsuji, M. Caricato, X. Li, H. P. Hratchian, A. F. Izmaylov, J. Bloino, G. Zheng, J. L. Sonnenberg, M. Hada, M. Ehara, K. Toyota, R. Fukuda, J. Hasegawa, M. Ishida, T. Nakajima, Y. Honda, O. Kitao, H. Nakai, T. Vreven, J. A. Montgomery, Jr., J. E. Peralta, F. Ogliaro, M. Bearpark, J. J. Heyd, E. Brothers, K. N. Kudin, V. N. Staroverov, R. Kobayashi, J. Normand, K. Raghavachari, A. Rendell, J. C. Burant, S. S. Iyengar, J. Tomasi, M. Cossi, N. Rega, J. M. Millam, M. Klene, J. E. Knox, J. B. Cross, V. Bakken, C. Adamo, J. Jaramillo, R. Gomperts, R. E. Stratmann, O. Yazyev, A. J. Austin, R. Cammi, C. Pomelli, J. W. Ochterski, R. L. Martin, K. Morokuma, V. G. Zakrzewski, G. A. Voth, P. Salvador, J. J. Dannenberg, S. Dapprich, A. D. Daniels, O. Farkas, J. B. Foresman, J. V. Ortiz, J. Cioslowski and D. J. Fox, Gaussian09 Revision D.01, Gaussian Inc., Wallingford CT, 2009.

81 M. Meuwly and J. M. Hutson, J. Chem. Phys., 1999, 110, 8338-8347.

82 P. M. Gill, B. G. Johnson, J. A. Pople and M. J. Frisch, Chem. Phys. Lett., 1992, 197, 499-505.

83 R. Parr and W. Yang, Density-Functional Theory of Atoms and Molecules, Oxford University Press, USA, 1989.

84 M. Head-Gordon, J. A. Pople and M. J. Frisch, Chem. Phys. Lett., 1988, 153, 503-506.

85 M. J. Frisch, M. Head-Gordon and J. A. Pople, Chem. Phys. Lett., 1990, 166, 281-289.

86 M. J. Frisch, M. Head-Gordon and J. A. Pople, Chem. Phys. Lett., 1990, 166, 275-280.

87 M. Head-Gordon and T. Head-Gordon, Chem. Phys. Lett., 1994, 220, 122-128.

88 B. R. Brooks, C. L. Brooks, III, A. D. Mackerell, Jr., L. Nilsson, R. J. Petrella, B. Roux, Y. Won, G. Archontis, C. Bartels, S. Boresch, A. Caflisch, L. Caves, Q. Cui, A. R. Dinner, M. Feig, S. Fischer, J. Gao, M. Hodoscek, W. Im, K. Kuczera, T. Lazaridis, J. Ma, V. Ovchinnikov, E. Paci, R. W. Pastor, C. B. Post, J. Z. Pu, M. Schaefer, B. Tidor, R. M. Venable, H. L. Woodcock, X. Wu, W. Yang, D. M. York and M. Karplus, J. Comput. Chem., 2009, 30, 1545-1614.
89 B. Brooks, R. Bruccoleri, D. Olafson, D. States, S. Swaminathan and M. Karplus, J. Comput. Chem., 1983, 4, 187-217.

90 A. MacKerel Jr., C. Brooks III, L. Nilsson, B. Roux, Y. Won and M. Karplus, CHARMM: The Energy Function and Its Parameterization with an Overview of the Program, John Wiley \& Sons, Chichester, 1998, vol. 1, pp. 271-277.

91 Y. Yang, H. Yu, D. York, Q. Cui and M. Elstner, J. Phys. Chem. A, 2007, 111, 10861-10873.

92 D. Riccardi, P. Schaefer, Y. Yang, H. Yu, N. Ghosh, X. PratResina, P. Konig, G. Li, D. Xu, H. Guo, M. Elstner and Q. Cui, J. Phys. Chem. B, 2006, 110, 6458-6469.

93 D. A. McQuarrie, Statistical Mechanics, University Science Books, 1st edn, 2000.

94 M. Schmitz and P. Tavan, J. Chem. Phys., 2004, 121, 12233-12246.

95 R. Ramirez, T. Lopez-Ciudad, P. Kumar and D. Marx, J. Chem. Phys., 2004, 121, 3973-3983.

96 S. A. Egorov, K. F. Everitt and J. L. Skinner, J. Phys. Chem. A, 1999, 103, 9494-9499.

97 M. Schmitz and P. Tavan, J. Chem. Phys., 2004, 121, 12247-12258.

98 D. L. Howard and H. G. Kjaergaard, J. Phys. Chem. A, 2006, 110, 10245-10250.

99 Y.-L. Cheng, H.-Y. Chen and K. Takahashi, J. Phys. Chem. A, 2011, 115, 5641-5653.

100 K. L. Plath, K. Takahashi, R. T. Skodje and V. Vaida, J. Phys. Chem. A, 2009, 113, 7294-7303.

101 E. B. Wilson, Phys. Rev., 1934, 45, 706-714.

102 M. L. Sage and J. Jortner, Bond Modes, John Wiley \& Sons, Inc., 2007, pp. 293-322.

103 J. Vazquez and J. F. Stanton, Mol. Phys., 2006, 104, 377-388.

104 H.-J. Werner, P. J. Knowles, G. Knizia, F. R. Manby, M. Schütz, P. Celani, T. Korona, R. Lindh, A. Mitrushenkov, G. Rauhut, K. R. Shamasundar, T. B. Adler, R. D. Amos, A. Bernhardsson, A. Berning, D. L. Cooper, M. J. O. Deegan, A. J. Dobbyn, F. Eckert, E. Goll, C. Hampel, A. Hesselmann, G. Hetzer, T. Hrenar, G. Jansen, C. Köppl, Y. Liu, A. W. Lloyd, R. A. Mata, A. J. May, S. J. McNicholas, W. Meyer, M. E. Mura, A. Nicklass, D. P. O’Neill, P. Palmieri, D. Peng, K. Pflüger, R. Pitzer, M. Reiher, T. Shiozaki, H. Stoll, A. J. Stone, R. Tarroni, T. Thorsteinsson and M. Wang, MOLPRO, version 2012.1, a package of ab initio programs, 2012.

105 O. Guvench, S. S. Mallajosyula, E. P. Raman, E. Hatcher, K. Vanommeslaeghe, T. J. Foster, F. W. Jamison, II and A. D. MacKerell, Jr., J. Chem. Theory Comput., 2011, 7, 3162-3180.

106 P. Durlak and Z. Latajka, Chem. Phys. Lett., 2009, 477, 249-254.

107 K. Giese, M. Petković, H. Naundorf and O. Kühn, Phys. Rep., 2006, 430, 211-276.

108 F. Hedin, K. elHage and M. Meuwly, J. Chem. Inf. Model., 2016, DOI: 10.1021/acs.jcim.6b00280. 